\title{
Quantification of Patient Specific Dosimetry in Radionuclide Therapy:
}

\author{
A Phantom Study
}

\author{
Kamila Afroj Quadir ${ }^{1}$, Brian Zimmermann², Md. Nahid Hossain ${ }^{1}$, Md. Nurul Islam ${ }^{1}$ \\ Ferdoushi Begum ${ }^{1}$, Tanvir Ahmed Biman', Mohammad Anwar-Ul-Azim ${ }^{1}$ \\ ${ }^{1}$ National Institute of Nuclear Medicine \& Allied Sciences, BAEC, BSM Medical University Campus, Shahbagh, Dhaka, \\ Bangladesh. ${ }^{2}$ Physics Laboratory, National Institute of Standards and Technology, MD, USA.
}

Address For Correspondence: Prof. Kamila Afroj Quadir, National Institute of Nuclear Medicine \& Allied Sciences, BAEC, BSM Medical University Campus, Shahbagh, Dhaka, Bangladesh. Email: q.kamila@gmail.com

\begin{abstract}
The accuracy of patient specific dosimetry is correlated with measured organ activity by gamma camera and SPECT system. The assessment of the radiation-absorbed dose by patients undergoing nuclear medicine investigation requires accurate measurement of organ activity, biokinetics data, as well as physical data. Activities were estimated by using Ba-133 phantom with both planar and SPECT systems. The objective of the study was to measure the activities of Ba-133 from gamma camera images using both planar and SPECT studies and compare the reference values with the dose calibrator values to quantify the actual activity with gamma camera. Four Ba-133 sources of different volume and activity 379, 950, 1219 and $1150 \mathrm{KBq}$ are measured by using Veenstra Instrument VDC 404 Dose Calibrator. The second smallest source was used to determine the calibration factor. Acquisition, corrections, reconstruction, quantification and measuring activity from both planar and SPECT imaging were done with all Ba-133 sources in air. The activities of the Ba-133 sources were also measured using I-131 settings of the dose calibrator. The measurement of the second smallest source was used to obtain the calibration factor. This calibration factor was used to convert the planer and SPECT image count of all the sources into activities. In case of both planar and SPECT gamma camera, the measurements showed good correlations and all the values varied within $\pm 15 \%$. Planer and SPECT gamma camera image counts can be used to calculate activity in the organ. This information can play a very significant role in evaluating image based patient specific dosimetry in radionuclide therapy.
\end{abstract}

Keywords: Patient specific dosimetry, Radionuclide therapy, Ba-133 phantom

\section{INTRODUCTION}

For the assessment of the radiation absorbed dose by patients undergoing nuclear medicine investigations, accurate measurement of organ activity, biokinetics data, as well as physical data e.g. decay properties of the radionuclide, emitted radiation and source-target geometry are required. Measurement of organ activity is very important for the calculation of absorbed dose for the purpose of patient specific dosimetry. From the very beginning, the model based approach (1-3), which has been developed by the MIRD Committee of the society of nuclear medicine as MIRD Scheme and has been used for the calculation of internal dosimetry as a fundamental tool.

With the advancement of nuclear medicine equipment the technology is now moving towards the image based dosimetry (4-5). This technique is assumed to be more accurate.

The objective of the study was to measure the activity of Ba-133 phantom from gamma camera images using both planar and SPECT studies and compare the reference values with the dose calibrator values.

\section{MATERIALS AND METHODS}

Four Ba-133 (H.L=10.74 years and $\mathrm{E}=356 \mathrm{KeV}$ ) cylindrical sources of different volume and activity were used and arranged in a Jaszcjack Phantom as shown in figure 1 (A) and 1 (B) respectively. The sources specifications are given in the table 1 , table 2 and table 3 shows the activity concentration in each rod (Ba-133) at a reference time in 1 July 2011. 

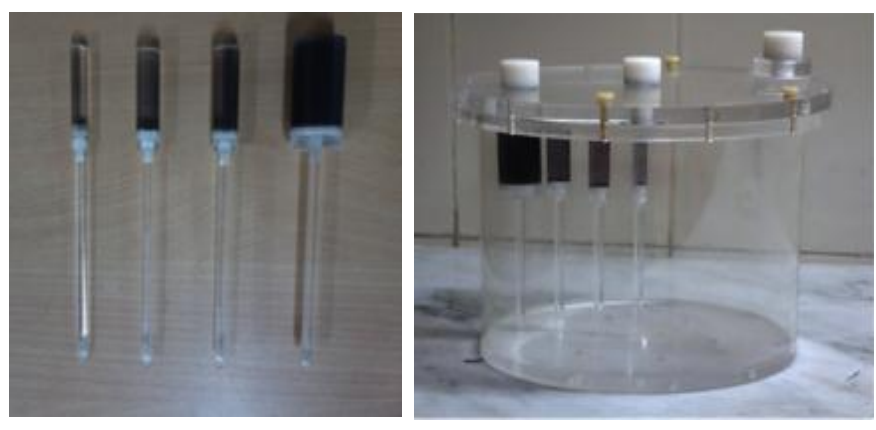

Figure 1: (A). Ba-133 sources, (B) Arrangement of the Ba-133 sources in a Jaszcjack Phantom

Table 1: The diameter and size of $\mathrm{Ba} \mathbf{- 1 3 3}$ sources.

\begin{tabular}{|l|c|c|}
\hline \multicolumn{1}{|c|}{ Source Size } & Diameter $\mathbf{( c m )}$ & Volume $\left.\mathbf{( c m}^{3}\right)$ \\
\hline Rod-1 smallest & 1 & 1.9 \\
\hline Rod 2 & 1.4 & 4.8 \\
\hline Rod 3 & 1.6 & 6.1 \\
\hline Rod-4 Largest & 3 & 24.1 \\
\hline
\end{tabular}

Table 2: The activity of sources at reference date in July 2011 (I-131 settings).

\begin{tabular}{|c|c|}
\hline Parameters & Activity in KBq \\
\hline Rod 1(smallest) & 1209 \\
\hline Rod 2 & 3005 \\
\hline Rod 3 & 3840 \\
\hline Rod 4 (largest) & 3486 \\
\hline
\end{tabular}

Table 3: Activity concentration in each rod (Ba133) at reference time (I-131 settings).

\begin{tabular}{|c|c|}
\hline Parameters & Activity concentration $\mathbf{K B q} / \mathbf{m L}$ \\
\hline Rod 1(smallest) & 636.32 \\
\hline Rod 2 & 626.04 \\
\hline Rod 3 & 629.51 \\
\hline Rod 4 (largest) & 144.65 \\
\hline
\end{tabular}

The second smallest Ba-133 source was used as calibration source for measuring the sensitivity of the gamma camera. The sources received from National Institute of Standards and Technology (NIST), USA, in October 2011.

A Veenstra Instruments dose calibrator was used (Model: VDC 404) to measure the activity of the sources of both Ba-133 and I-131 settings (Table 4).
Table 4: Activity measurement with dose calibrator in both Ba-133 and I-131 settings

\begin{tabular}{|l|c|c|}
\hline \multirow{2}{*}{ Source } & \multicolumn{2}{|c|}{ Activity (kBq) } \\
\cline { 2 - 3 } & Setting: Ba-133 & Setting: I-131 \\
\hline Rod 1 - smallest & 379 & 1111 \\
\hline Rod 2 & 950 & 2760 \\
\hline Rod 3 & 1219 & 3527 \\
\hline Rod 4 -largest & 1150 & 3202 \\
\hline
\end{tabular}

The Jaszcjack phantom with the sources mounted in the leveled couch was placed as shown in figure 2 (A) and 2 (B). Siemens Medical Soln.inc, ECAM (dual head) gamma camera for planar imaging was used for the acquisition.

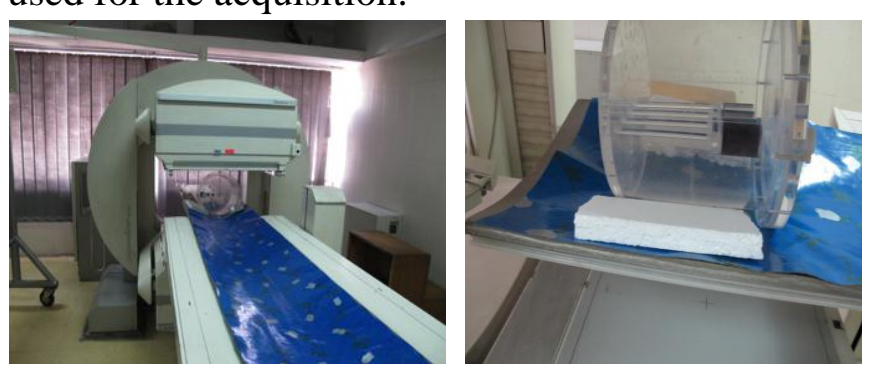

Figure 2: (A). The phantom leveled on the couch, (B) Position of the sources.

The Software version was 6.5.9-12-VE3/FOSR 3.4 and crystal thickness was $9.5 \mathrm{~mm}$ high energy parallel hole collimator, matrix size: 256 X 256 and pixel size: $2.42 \mathrm{~mm}$ were used as acquisition parameters. The acquisition time was $1000 \mathrm{sec}$ with $15 \%$ energy window. The estimated energy resolution for ${ }^{99 \mathrm{~m}} \mathrm{Tc}$ (at $140 \mathrm{keV}$ ) was 8.75 and background count rate (for $1000 \mathrm{sec}$ ) was $0.71 / \mathrm{pixel}$.

For SPECT imaging the same equipment was used. Dual head gamma cameras were used for the SPECT acquisition. Matrix size 128 X 128 and pixel size $4.84 \mathrm{~mm}$ were used as acquisition parameters. The acquisition time per view was 60 second. The background count rate was detected at $0.00056 \mathrm{cps} /$ voxel. No transmission/CT parameters were used for attenuation correction. The processing parameters that were chosen for the 
imaging for planer and SPECT are given in table 5 and 6 respectively. Each test was done thrice.

Table 5: The processing parameters for planar imaging

\begin{tabular}{|c|c|c|c|}
\hline Parameters & Test 1 & Test 2 & Test 3 \\
\hline $\begin{array}{l}\text { Sensitivity (calculated } \\
\text { using second smallest } \\
\text { source activity from } \\
\text { source measured with dose } \\
\text { calibrator at I-131 setting, } \\
\text { time: } 10 \text { min) }\end{array}$ & $10.16 \mathrm{cps} / \mathrm{MBq}$ & $9.68 \mathrm{cps} / \mathrm{MBq}$ & $9.92 \mathrm{cps} / \mathrm{MBq}$ \\
\hline $\begin{array}{l}\text { ROI(Number of pixels in } \\
\text { each region) }\end{array}$ & $\begin{array}{l}\text { i-383, ii-380, } \\
\text { iii-386, iv-398 }\end{array}$ & $\begin{array}{l}\text { i-376, ii-370, } \\
\text { iii-375, iv-386 }\end{array}$ & $\begin{array}{l}\text { i-382, ii- } 385, \\
\text { iii-383, iv-402 }\end{array}$ \\
\hline Background correction & Done & Done & Done \\
\hline
\end{tabular}

Table 6: The processing parameters for SPECT imaging

\begin{tabular}{|l|l|l|l|}
\hline Parameters & \multicolumn{1}{|c|}{ Test 1 } & \multicolumn{1}{c|}{ Test 2 } & \multicolumn{1}{c|}{ Test 3 } \\
\hline $\begin{array}{l}\text { Voxel value } \\
\text { to activity } \\
\text { conversion } \\
\text { factor }\end{array}$ & $\begin{array}{l}21.85 \mathrm{cps} / \mathrm{MBq} \& \\
0.005 \mathrm{cps} / \mathrm{MBq} \\
\text { per voxel }\end{array}$ & $\begin{array}{l}22.49 \mathrm{cps} / \mathrm{MBq} \& \\
0.005 \mathrm{cps} / \mathrm{MBq} \\
\text { per voxel }\end{array}$ & $\begin{array}{l}22.84 \mathrm{cps} / \mathrm{MBq} \\
\& \\
0.005 \mathrm{cps} / \mathrm{MBq} \\
\text { per voxel }\end{array}$ \\
\hline $\begin{array}{l}\text { Method used } \\
\text { to estimate } \\
\text { conversion } \\
\text { factor }\end{array}$ & $\begin{array}{l}\text { 2nd smallest source was used as calibration source and then } \\
\text { conversion factor has been calculated from data calculation } \\
\text { with tomographic reconstruction }\end{array}$ \\
\hline $\begin{array}{l}\text { Number of } \\
\text { voxels in } \\
\text { each region }\end{array}$ & $\begin{array}{l}\text { Sources: } \\
\text { i-4674, ii-4498, } \\
\text { iii-4498 \& } \\
\text { iv-4763 }\end{array}$ & $\begin{array}{l}\text { Sources: } \\
\text { i-4582, ii-4359, } \\
\text { iii-4465 \& } \\
\text { iv-4642 }\end{array}$ & $\begin{array}{l}\text { Sources: } \\
\text { i-4586,ii-4476, } \\
\text { iii-4478 \& } \\
\text { iv-4732 }\end{array}$ \\
\hline $\begin{array}{l}\text { Background } \\
\text { correction }\end{array}$ & $0.00056 \mathrm{cps} / \mathrm{voxel}$ & $\begin{array}{l}0.00049 \\
\text { cps/voxel }\end{array}$ & 0.00053 cps/voxel \\
\hline $\begin{array}{l}\text { Method used } \\
\text { for activity } \\
\text { concentration } \\
\text { measurement }\end{array}$ & activity/ volume & activity/ volume & activity/ volume \\
\hline
\end{tabular}

The sources were placed parallel inside the Jaszcjack Phantom (as shown in figure1) under the gamma camera at a distance $5 \mathrm{~cm}$ for planar imaging. For SPECT imaging the source was placed at $20 \mathrm{~cm}$ Radius of Rotation (ROR). To minimize the statistical noise, data were acquired 3 times for each processing setting.

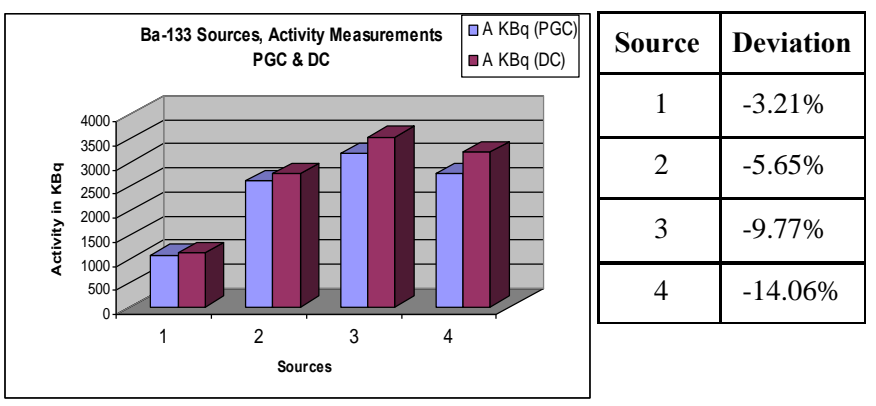

Figure 3: Activity measurement from planar Gamma Camera (PGC) and dose calibrator (DC).

For SPECT imaging, the tomographic reconstruction was done with the cut-off frequency 0.4 , order 5 and Butterworth filter.

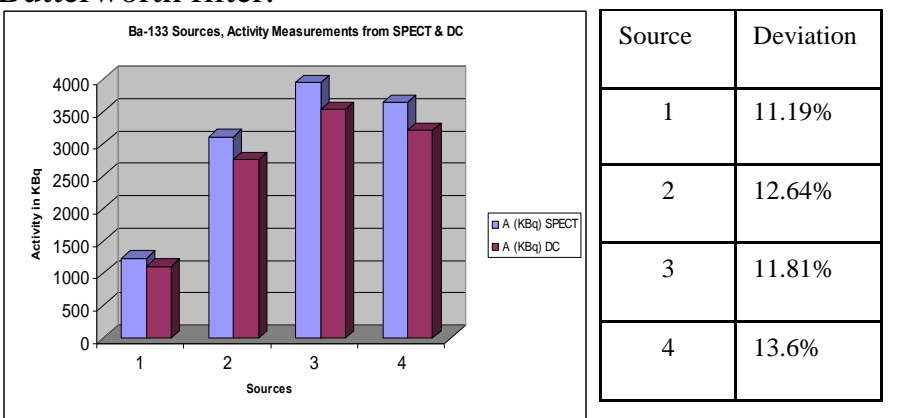

Figure 4: Activity measurement from SPECT and dose calibrator (DC).

\section{RESULTS AND DISCUSSION}

The activity of Ba-133 was measured in dose calibrator at I-131 settings. Imaging in gamma camera was also performed at I-131 settings. Because the energy of both Ba-133 and I-131 are close, the activity of the four sources were measured at $1111,2760,3527$ and $3202 \mathrm{KBq}$ with I-131 settings by using dose calibrator. The second smallest source, $2760 \mathrm{KBq}$, was used as calibration source for measuring the sensitivity of the gamma camera as well as for calibration factor. The measurement of the source activity was done by using Siemens ECAM gamma camera and SPECT system. This calibration factor was then used to convert the planar and SPECT image count of all the sources into activities. For both planar and SPECT gamma camera, the measurements showed good correlations and all values varied within $\pm 15 \%$ (Figure 3 and figure 4). 
Quantitative Imaging, such as, acquisition, corrections, reconstruction, quantification and measuring activity from both planar and SPECT imaging was done with all Ba-133 sources.

Due to the limitation of the equipment the Transmission/CT parameters were absent in the camera. Furthermore, scatter correction could not be performed as the software did not support the process. However, if these procedures were carried out the result quality would further improve.

The internal dose quantifications are superior to conventional dose calculation and should therefore be the future direction of the development of dosimetry development in radionuclide therapy.

Imaging equipment can now be used as a guide in determining a more accurate dosimetry, which is patient specific and therefore will result in optimization of therapeutic benefit.

\section{CONCLUSION}

Planar and SPECT gamma camera image counts can therefore be used to calculate activity in organ. The dose from the source can be measured by calculating activity of the image taken by the planner gamma camera and SPECT. This information can play a very significant role in evaluating image based patient specific dosimetry in radionuclide therapy. Images from SPECT/ CT and PET/ CT would give even better result.

\section{ACKNOWLEDGEMENT}

This work was partly carried out within the framework of the International Atomic Energy Agency's (IAEA) CRP Project Program "Development of Quantitative Nuclear Medicine Imaging for Optimized Patient Specific Dosimetry (E2.10.07)".

\section{REFERENCES}

1. 1. Jonsson L, Liu X, Jonsson B-A, et al. A dosimetry model for the small intestine incorporating intestinal wall activity and cross-dosses. J Nucl Med 2002; 43:16571664.

2. Loevinger $\mathrm{R}$. The MIRD perspective. In: Adelstein S, Kassis A, Burt R, eds. Dosimetry of administrated radionuclides. Washington, DC: American college of Nuclear Physicians/Department of Energy1989:29-43.

3. Lovinger R, Budinger TF, Watson EE. MIRD Primer for Absorbed Dose Calculations. Revised. New York, NY: Society of Nuclear Medicine.

4. Zaidi H, Quantitative Analysis in Nuclear Medicine Imaging $1^{\text {st }}$ ed. USA: Institute of Physics publishing 2003:263.

5. Quadir KA, Zimmermann B, Hossain MN, Begum F, Islam $\mathrm{MN}$ and Biman TA. Image based patient specific dosimetry in radionuclide therapy: a phantom study. Abstract. Bangladesh Cancer Congress 2014. 